\title{
Ion beam irradiation of polyglactin mesh for improving biocompatibility
}

\author{
Toshiyuki Tanaka ${ }^{1,2, *}$, Hirofumi Yajima ${ }^{2}$, \\ Hiroshi Ujiie $^{3}$, Yoshiaki Suzuki ${ }^{1}$ and Akira Fukutome ${ }^{4}$
}

\author{
1. RIKEN, 2-1 Hirosawa, Wako-shi, Saitama 351-0198, Japan \\ 2. Tokyo University of Science, 1-3 Kagurazaka, Shinjuku-ku, Tokyo 162-8601, Japan \\ 3. Tokyo Rosai Hospital, 4-13-21 Omoriminami, Ota-ku, Tokyo 143-0013, Japan \\ 4. Zeon Medical Inc., 422-1 Futagamishin, Takaoka-shi, Toyama 933-0981, Japan \\ *Corresponding author. Tel.: +81-48-462-5079, Fax: +81-48-462-5004 \\ E-mail: tttanaka@riken.jp (Toshiyuki Tanaka)
}

Polyglactin is a bioadsorbable polymer used as tissue engineering scaffolds. However, it has poor cell attachment properties. In this study, polyglactin mesh is irradiated with $\mathrm{Kr}^{+}$to improve their biocompatibility. $\mathrm{Kr}^{+}$-irradiation was performed into the mesh at an energy of $50 \mathrm{keV}$ with a fluence of $1 \times 10^{13}, 1 \times 10^{14}$ and $1 \times 10^{15}$ ions $/ \mathrm{cm}^{2}$, respectively. After the irradiation, the changes of original chemical bonds and the production of new functional groups were measured by Fourier transform infrared spectroscopy (FT-IR-ATR). Hydrolytic degradation of irradiated meshes was carried out in phosphate buffer solution (PBS). In animal studies, these meshes were wrapped around the carotid arteries of rabbits. Fibroblast cells (L929) were seeded on non-irradiated and irradiated meshes. The scission of original chemical bonds was caused by ion beam irradiation with increasing fluences. Irradiated meshes degraded in the same manner as non-irradiated. Seeded cells attached and spread well on irradiated meshes compared with non-irradiated. Irradiated mesh adhered well and inhibited the inflammation and thickening of the vascular wall. Irradiated polyglactin meshes exhibit excellent biocompatibility and retain its biodegradability. Thus, ion beam irradiated polyglactin will be useful for clinical applications.

Key words: Polyglactin, Ion beam irradiation, Hydrolytic degradation, Fibroblasts, Animal study

\section{INTRODUCTION}

Poly(lactide), poly(glycolide) and their copolymers (polyglactin) have been used as sutures, materials for drug delivery systems, and scaffolds for tissue engineering applications. These polymers are non-toxic with respect to cells, since they are hydrolytically resolved into metabolic products via metabolic pathways and subsequently removed from the body. Tissue engineering scaffolds prepared from these biodegradable polymers were adsorbed during the regeneration of damaged or missing tissues. Polyglactin attracted much attention for use in biomaterials since it is FDA approved for clinical applications. The important properties of biodegradable scaffolds are those concerning the mechanical performance, degradation rate and biocompatibility. The mechanical properties and degradation rate are controlled by varying the proportion of lactide to glycolide on polyglactin scaffolds [1-3]. However, its poor cell affinity caused many problems, owing to the lack of cell binding sites and functional groups. Many researchers have improved the cell affinity of biodegradable scaffolds [4-6].

Previous studies demonstrated that ion beam irradiation into PLLA surface was a powerful method for cell attachment [7-8]. New functional groups and new carbon structure induced by ion beam irradiation possibly have great effects on the improvement of cell attachment. In vivo animal studies of irradiated segmented polyurethane (SPU) [9], silicone [10] and ePTFE [11-13] exhibited excellent biocompatibility. The biocompatibility of biodegradable polymers has not yet been investigated precisely. In this work, the tissue compatibility of irradiated polyglactin meshes was evaluated.

\section{EXPERIMENTAL}

\subsection{Materials}

Polyglactin mesh in the form of a sterile knitted sheet (VICRYL* Woven Mesh) was purchased from ETHICON, J\&J, USA. The mesh 
is $90 / 10$ copolymer of glycolide and lactide acids.

\subsection{Ion beam irradiation}

Polyglactin mesh was cut into $2.0 \mathrm{~cm} \times 2.0 \mathrm{~cm}$ pieces and used as a substrate. $\mathrm{Kr}^{+}$-irradiation was performed on the specimens at an energy of $50 \mathrm{keV}$ with fluences of $1 \times 10^{13}, 1 \times 10^{14}$ and $1 \times 10^{15}$ ions $/ \mathrm{cm}^{2}$. The beam current density was kept at $0.05 \mu \mathrm{A} / \mathrm{cm}^{2}$ to prevent heating of the specimens.

\subsection{Characterization}

The scission of original chemical bonds and production of new functional groups induced by ion beam irradiation were investigated by Fourier transform infrared spectroscopy with attenuated total reflectance (Nexus 470: Thermo Nicolet, USA). The analysis was conducted with an internal reflection element $\left(\mathrm{Ge}, 45^{\circ}\right.$ incident angle) and a resolution of $4 \mathrm{~cm}^{-1}$. Each spectrum was obtained after 64 scans from 1000 to 4000 $\mathrm{cm}^{-1}$.

\subsection{Hydrolytic degradation test}

For the hydrolytic degradation test, the effect of ion beam irradiation on the hydrolysis was investigated. Non-irradiated and irradiated meshes were placed on the bottom of the $60 \mathrm{~mm}$ tissue culture dishes, phosphate buffer solution $(\mathrm{pH}=7.4)$ was added to the dishes, and then these specimens were left standing at $37{ }^{\circ} \mathrm{C}$ in a $5 \% \mathrm{CO}_{2}$ environment. These samples were taken out from the degradation medium, and dried at room temperature. Morphological changes of these meshes were observed with a scanning electron microscope (SEM: JSM6330F, JEOL, Japan) after $\mathrm{Au}$ evaporation.

\subsection{Cell culture}

Mouse fibroblast cells (L929: RIKEN CELL BANK, Japan) were maintained in a culture medium (RPMI 1640: Nissui Pharm. Co., Japan) supplemented with $10 \%$ fetal bovine serum (FBS: Sanko-jyuyaku Co., Japan). All samples were sterilized with UV radiation for $10 \mathrm{~min}$ on both sides. Non-irradiated and irradiated meshes were cut into $1 \mathrm{~cm} \times 1 \mathrm{~cm}$ pieces and placed on the bottom of the 24-well tissue culture plates. Suspensions of L929 cells $\left(1 \times 10^{5}\right.$ cells $\left./ \mathrm{ml}\right)$ were seeded onto the substrates and incubated at $37{ }^{\circ} \mathrm{C}$ in a humidified atmosphere with $5 \% \mathrm{CO}_{2}$. After 5 days of incubation, the culture medium was removed, rinsed three times by $\mathrm{PBS}$ solution and fixed in $2.5 \%$ glutaraldehyde for $2 \mathrm{~h}$ at $37^{\circ} \mathrm{C}$ in a $5 \% \mathrm{CO}_{2}$ environment, dehydrated through a series of graded ethanol solution, and then air-dried overnight. The samples were sputter coated, and observed by SEM.

\subsection{Animal study}

Japanese white rabbits weighing 3 to $4 \mathrm{~kg}$ were used in this study. Non-irradiated and irradiated meshes were wrapped around the carotid arteries with fibrin glue (Bolheal,
Kaketsuken, Japan) under sodium pentobarbital anesthesia (Somnopetyl, Kyoritsu Seiyaku Co., Japan) (Fig. 1). All of the procedures were performed under sterile conditions. Recipients of the meshes were sacrificed at 2 and 4 weeks, and implanted specimens were surgically excised under anesthesia. The implants were fixed in $10 \%$ buffered formalin and thin sections were cut from paraffin-embedded tissue. Histological sections were stained with haematoxylin and eosin. Tissue adhesive properties of non-irradiated and irradiated meshes with respect to the vascular wall were investigated.

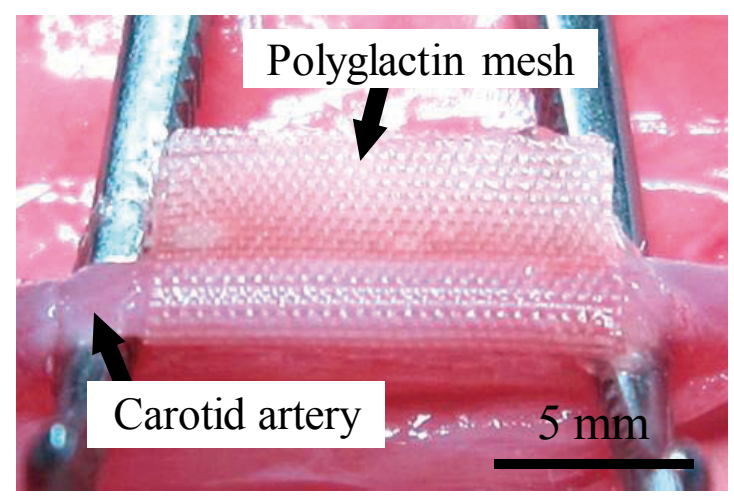

Fig. 1. Macroscopic view of irradiated mesh wrapped around the carotid arteries of rabbits.

\section{RESULTS AND DISCUSSION}

\subsection{Characterization}

In FT-IR-ATR spectra, typical peaks for $\mathrm{C}=\mathrm{O}$, $\mathrm{C}-\mathrm{O}$ stretching and $\mathrm{C}-\mathrm{O}-\mathrm{C}$ groups were observed at 1750,1134 , and $1080 \mathrm{~cm}^{-1}$, respectively (Fig. 2). Another peak about $1450 \mathrm{~cm}^{-1}$ is due to $\mathrm{C}-\mathrm{H}$ stretching in methyl groups $[14,15]$. These peaks' intensities decreased with increasing fluence. The production of new functional groups and the peak shift of $1750 \mathrm{~cm}^{-1}$ towards lower wavenumber were not observed. This is because the accelerated energy is relatively low and the energy of $\mathrm{Kr}^{+}$ions was reduced, mainly via nuclear interaction with polyglactin meshes.

\section{2. Hydrolytic degradation test}

Figure 3 depicts SEM photographs of degraded non-irradiated and irradiated meshes at 4 weeks. The fibers of the meshes were cracked in all the specimens. Irradiated mesh with a fluence of $1 \times 10^{13}$ ions $/ \mathrm{cm}^{2}$ showed no significant morphological change in comparison with non-irradiated. With a fluence of $1 \times 10^{14}$ ions $/ \mathrm{cm}^{2}$, large cracks were observed in the fibers owing to radiation damage. With a fluence of $1 \times 10^{15}$ ions $/ \mathrm{cm}^{2}$, the exfoliation of the irradiated layer was observed due to the large amount of radiation damage. This phenomenon is similar to the case of high energy ion beam irradiation into PLLA sheets $[6,16,17]$. It is considered that ion beam irradiation forms void space between the irradiated layer and substrates, resulting in the 
exfoliation of the irradiated layer in PBS solution It is important that the irradiated layer is maintained on the material surface until the regeneration of the damaged or missing tissues. From these experimental results, irradiated PLGA meshes maintained the hydrolysis ability and the suitable fluence is below $1 \times 10^{14}$ ions $/ \mathrm{cm}^{2}$.

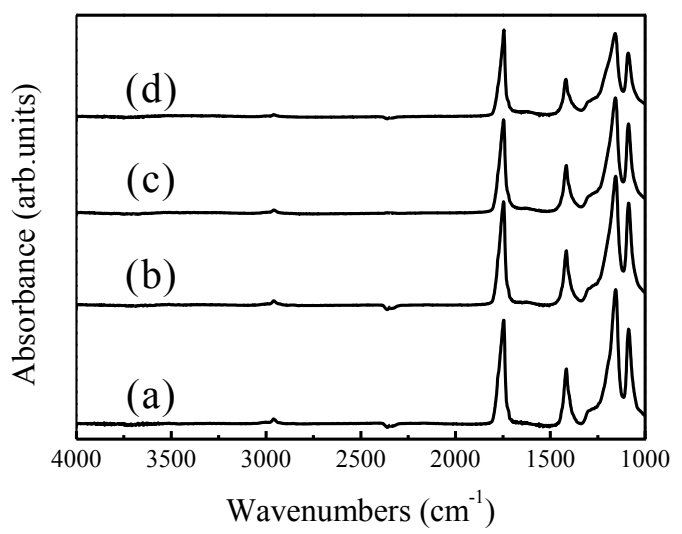

Fig. 2. FT-IR-ATR spectra of (a) non-irradiated and irradiated surface at an energy of $50 \mathrm{keV}$ with fluences of (b) $1 \times 10^{13}$, (c) $1 \times 10^{14}$ and (d) $1 \times 10^{15}$ ions $/ \mathrm{cm}^{2}$.
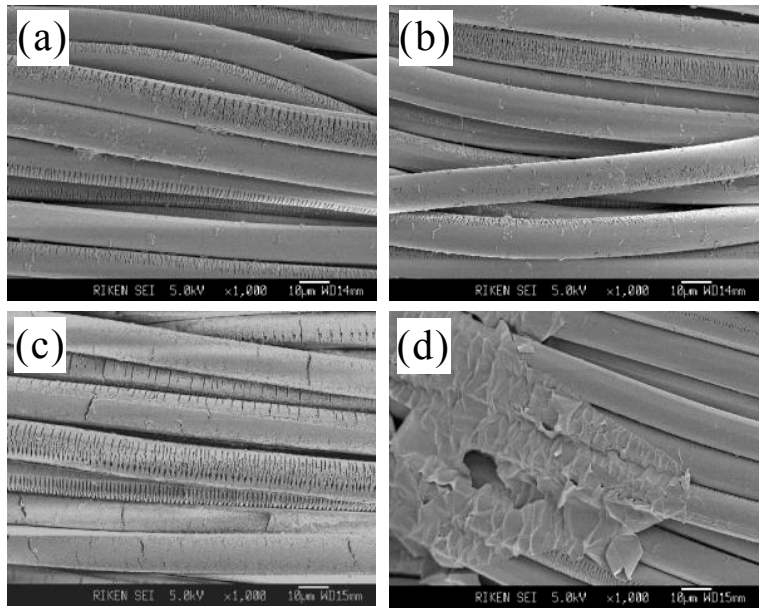

Fig. 3. Morphology of (a) non-irradiated and irradiated meshes with fluences of (b) $1 \times 10^{13}$, (c) $1 \times 10^{14}$ and (d) $1 \times 10^{15}$ ions $/ \mathrm{cm}^{2}$ after hydrolytic degradation for 4 weeks.

\section{3. Cell culture}

Figure 4 shows the attachment of L929 fibroblast cells seeded on non-irradiated and irradiated meshes with fluences of $1 \times 10^{13}$ and $1 \times 10^{14}$ ions $/ \mathrm{cm}^{2}$ after 5 days culture. A large number of round cells were observed on non-irradiated mesh. In contrast, the cells spread well on irradiated meshes. Cell attachment was dramatically improved with increasing fluence. Irradiated mesh with a fluence of $1 \times 10^{14}$ ions $/ \mathrm{cm}^{2}$ exhibited the best cell attachment and the specimen was used for the animal study.

\subsection{Animal study}

Figure 5 demonstrates the histology of the carotid artery wrapped by non-irradiated and irradiated meshes after implantation of 2 and 4 weeks. At 2 weeks, low biocompatibility of the non-irradiated mesh allowed the intrusion of some sort of cells and tissues between meshes and the blood vessel, resulting in thickening of the vascular wall. Meanwhile, irradiated mesh showed excellent tissue compatibility and depressed the thickening of an excess of cells growing around the blood vessel. Implanted non-irradiated and irradiated meshes were observed clearly at 2 weeks. At 4 weeks, the same tendency was observed in biocompatibility. Implanted meshes degraded and became thinner compared with those at 2 weeks. Both non-irradiated and irradiated meshes degraded in vivo. These results show that the biocompatibility of polyglactin mesh was dramatically improved by ion beam irradiation.
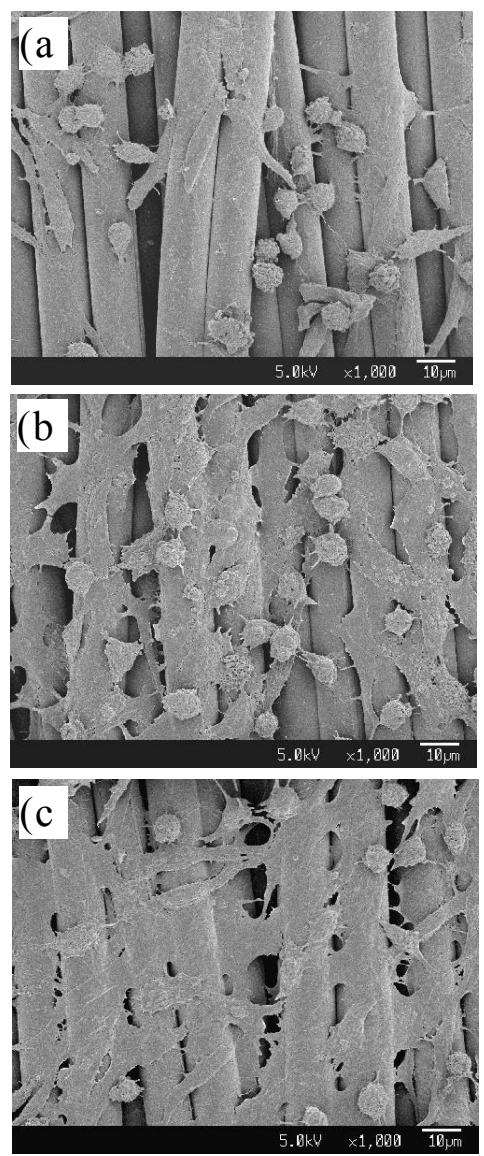

Fig 4. SEM photographs of cell attachment on (a) non-irradiated and irradiated surface with fluences of (b) $1 \times 10^{13}$ ions $/ \mathrm{cm}^{2}$ and (c) $1 \times 10^{14}$ ions $/ \mathrm{cm}^{2}$ at 5 days. 


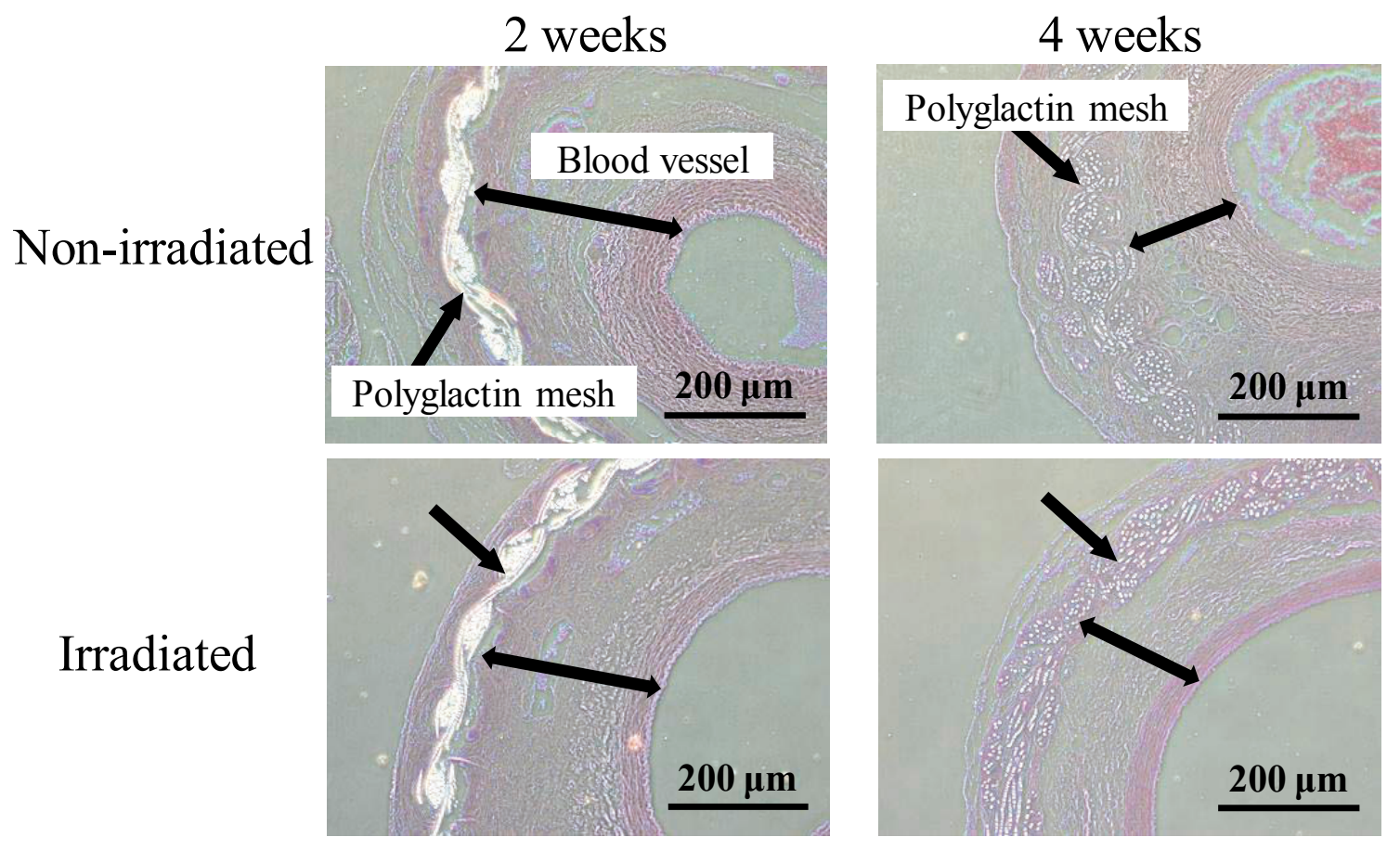

\section{CONCLUSION}

In this study, cell attachment and biocompatibility of polyglactin meshes were improved by ion beam irradiation. This is due to the breakage of chemical bonds and the subsequent carbonization. From the results of an in vitro cell culture and in vivo animal study, irradiated meshes have excellent biocompatibility and retain its biodegradability. Irradiated polyglactin meshes are expected to have potential utility in wound healing materials and wrapping materials for a wide-neck aneurysm.

\section{REFERENCES}

[1]X. Zong, S. Ran, D. Fang, B. S. Hsiao and B. Chu, Polymer, 44, 4959-67 (2003).

[2]L. Wu and J. Ding, Biomaterials, 25, 5821-30 (2004).

[3]X. Zong, H. Bien, C.-Y. Chung, L. Yin, D. Fang, B. S. Hsiao, B. Chu and E. Entcheva, Biomaterials, 26, 5330-38 (2005).

[4]C. -T. Lee and Y. -D. Lee, J. Mater. Sci.: Mater. Med., 17, 1411-20 (2006).

[5]R. A. Quirk, W. C. Chan, M. C. Davies, S. J. B. Tendler and K. M. Shakeshe, Biomaterials, 22, 865-72 (2001).

[6]W. He, Z. Ma, T. Yong, W. E. Teo and S. Ramakrishna, Biomaterials, 26, 7606-15 (2005).

[7]T. Yotoriyama, Y. Suzuki, M. Iwaki and T. Tsukamoto, Trans. Mater. Res. Soc. of Jpn., 28, 503-06 (2003).

[8]T. Tanaka, K. Uchida, H. Yajima, H. Toida, R. Ujiie and Y. Suzuki, Trans. Mater. Res. Soc. of Jpn., 32, 929-32 (2007).

[9]M. Iwaki, A. Nakao, M. Kaibara, H. Sasabe, S. Kaneko, H. Nakajima, Y. Suzuki, M. Kusakabe and T. Fujihana, Nucl. Instr. and Meth. in Phys. Res. B106, 618-23 (1995).
[10]Y. Suzuki, M. Kusakabe, H. Akiba, K. Kusakabe and M. Iwaki, Nucl. Instr. and Meth. in Phys. Res. B59/60, 698-704 (1991).

[11]Y. Suzuki, Nucl. Instr. and Meth. in Phys. Res. B206, 501-06 (2003).

[12]Y. Suzuki, M.Iwaki, S. Tani, G. Oohashi and M. Kamio, Nucl. Instr. and Meth. in Phys. Res. B206, 538-42 (2003).

[13]T. Uchida, H. Toida, R. Ujiie, Y. Suzuki, H. Ujiie, T. Hori and S. Ando, Trans. Mater. Res. Soc. of Jpn., 33, 1031-34 (2008).

[14]T. Paragkumar N, D. Edith and S. Jean-Luc, Appl. Surf. Sci., 253, 2758-64 (2006).

[15]A. C. Jayasuriya and N. A. Ebraheim, J. Mater. Sci.: Mater. Med., 20, 1637-44 (2009).

[16]T. Yotoriyama, T. Tsukamoto, Y. Suzuki and M. Iwaki, Nucl. Instr. and Meth. in Phys. Res. B206, 527-31 (2003).

[17]T. Yotoriyama, Y. Suzuki, T. Tsukamoto and M. Iwaki, Trans. Mater. Res. Soc. of Jpn., 29, 587-90 (2004).

(Received January 26, 2010; Accepted June 14, 2011) 